\title{
DUBINS' PROBLEM IS INTRINSICALLY THREE-DIMENSIONAL
}

\author{
DIRK MITTENHUBER
}

\begin{abstract}
In his 1957 paper [1] L. Dubins considered the problem of finding shortest differentiable arcs in the plane with curvature bounded by a constant $\kappa>0$ and prescribed initial and terminal positions and tangents. One can generalize this problem to non-euclidian manifolds as well as to higher dimensions (cf. [15]).

Considering that the boundary data - initial and terminal position $x_{0}, x_{1}$ and tangents $v_{0}, v_{1}$ - are genuinely three-dimensional, it seems natural to ask if the $n$-dimensional problem always reduces to the threedimensional case. In this paper we will prove that this is true in the euclidian as well as in the noneuclidian case. At first glance one might consider this a trivial problem, but we will also give an example showing that this is not the case.
\end{abstract}

\section{INTRODUCTION}

Dubins' problem in $\mathbb{R}^{n}$ can be formulated as follows: Given points $x_{0}, x_{1} \in$ $\mathbb{R}^{n}$ and two vectors $v_{0}, v_{1}$ of unit length, find a curve $\gamma:[0, L] \rightarrow \mathbb{R}^{n}$ such that

(i) $\gamma(s)$ is a differentiable curve, parameterized by arclength,

(ii) $\gamma$ has curvature $\kappa(s)$ almost everywhere, and the curvature is essentially bounded by some positive constant $\kappa_{0}:|\kappa(s)| \leq \kappa_{0}$ a.e.,

(iii) $\gamma$ satisfies the boundary conditions:

$$
\gamma(0)=x_{0}, \dot{\gamma}(0)=v_{0}, \gamma(L)=x_{1}, \dot{\gamma}(L)=v_{1},
$$

(iv) Among all possible arcs satisfying (i)-(iii), $\gamma$ has shortest length, i.e. $L$ is minimal.

One of the well-known interpretations of this problem is to think of a car moving with constant speed in the plane (or $n$-space) subject to the constraint that it cannot make arbitrarily sharp turns, see [17], for example. In three-space it is better to think of a plumber laying pipes, for driving along an optimal arc might be a roller coaster ride, cf. Figure 2. In that case one would want to control not only the terminal tangent but the terminal orientation.

Dubins proved that optimal arcs in $\mathbb{R}^{2}$ are concatenations of circular arcs (with constant curvature $\kappa_{0}$ ) and straight line segments. Moreover he proves

Fachbereich Mathematik, TU Darmstadt, Schloßgartenstr. 7, 64289 Darmstadt, Germany. E-mail: mittenhuber@mathematik.tu-darmstadt.de.

The author greatfully acknowledges the support by the Alexander von HumboldtFoundation.

Received by the journal August 7, 1997. Revised November 25, 1997. Accepted for publication December 12, 1997.

(C) Société de Mathématiques Appliquées et Industrielles. Typeset by IATEX. 

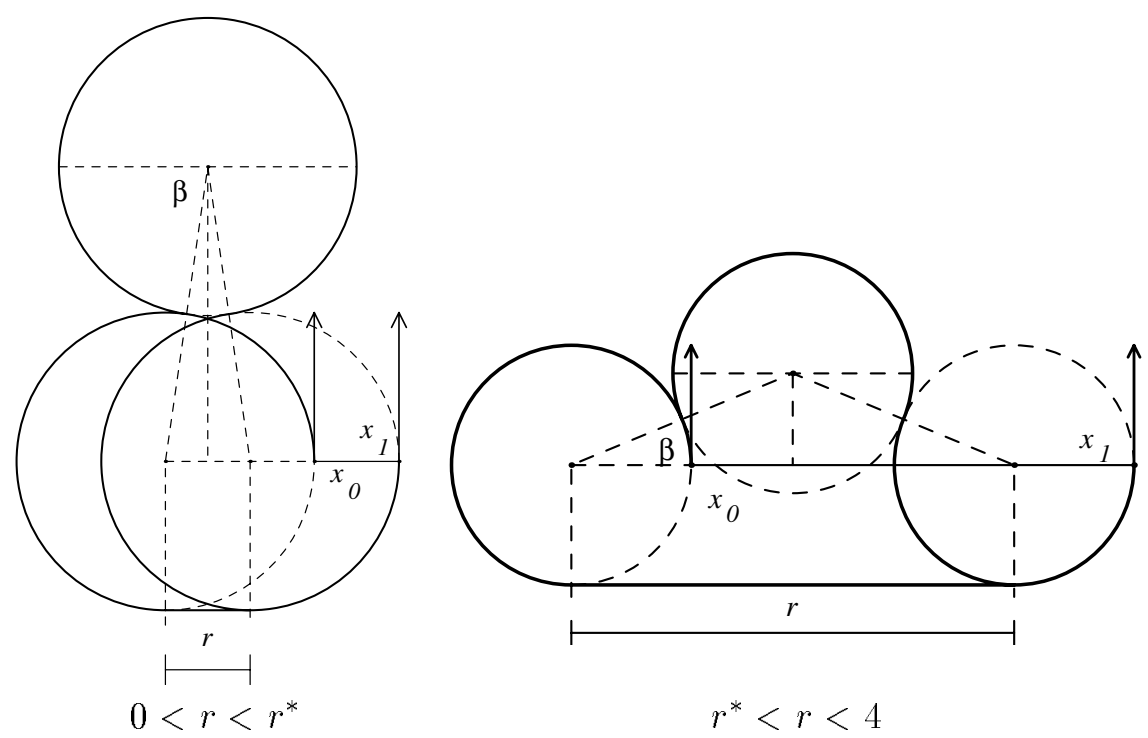

Figure 1. The optimal $r$-shift in $\mathbb{R}^{2}, r=4 \cos \beta$.

that optimal arcs consist of at most three pieces and that the line segment - if there is any - has to be in the middle, i.e. the optimal arcs follow what we now call Dubins' pattern, either $C C C$ or $C L C$. This reduces finding the optimal arcs to a finite problem. There are at most six candidates for optimal arcs. So all one has to do is determine these arcs and compare their lengths.

It was an open conjecture whether optimal arcs in $\mathbb{R}^{3}$ are again concatenations of circles and straight lines (cf. [17]) until this was disproved by Sussmann in [18].

In this paper Sussmann gave an example of a particular set of boundary conditions (or Dubins'data) $x_{0}, v_{0}, x_{1}, v_{1}$ and an arc $\gamma$ matching these data, such that any concatenation of circles and lines satisfying these boundary conditions must be strictly longer than $\gamma$. The arc $\gamma$ is a helicoidal arc, so it has constant curvature $\kappa$ but also constant torsion $\tau \neq 0$. And this example sheds a new light on the problem. The result is no longer surprising because thinking of the curvature as a cost functional in the variational problem, we are free to add torsion at no cost at all.

Considering Dubins' problem as an optimal control problem on the manifold $\mathbb{R}^{3} \times \mathbb{S}^{2}$ (position and unit tangent vector) Sussmann gives a detailed analysis of the structure of the extremals, i.e. curves satisfying the necessary conditions furnished by the Pontrjagin Maximum Principle (PMP). Of course Dubins' optimal arcs — concatenations of circles and lines — appear simply because the two-dimensional case is a subcase, but there is another type of extremals characterized by having constant curvature $\kappa_{0}$ and their torsion $\tau$ satisfying a certain ODE, see [18, p. 3308].

Passing to higher dimensions we observe that the boundary data $x_{0}, x_{1}$, $v_{0}, v_{1}$ are intrinsically three-dimensional because the vector space $V:=$ $\operatorname{span}\left\{x_{1}-x_{0}, v_{0}, v_{1}\right\}$ has dimension $\operatorname{dim}(V) \leq 3$, so the Dubins' data lie in the affine subspace $x_{0}+V$. And since the group $\mathrm{SE}(n)$ of euclidian motions ESAIM: COCV, FEBRUARY 1998, VOL. 3, 1-22 
of $\mathbb{R}^{n}$ obviously leaves the problem invariant - carrying optimal arcs into optimal arcs - it seems as if one could immediately reduce the problem to the subspace $V$. How could an arc leave this subspace and be shorter than an arc staying inside of $V$ ? But the attempts to prove this directly do not succeed.

So we look at the step from dimension 2 to 3 first, and suddenly we realize that this is a nontrivial question. We consider the following Dubins' data:

$$
x_{0}=0, \quad x_{1}=r e_{1}, \quad \text { with } 0<r \leq 4, \quad \text { and } v_{0}=v_{1}=e_{2} .
$$

Since we know the structure of optimal arcs in $\mathbb{R}^{2}$, it is not hard to verify that for small $r>0$ the optimal arc in $\mathbb{R}^{2}$ is of type $C L C$ and has length $L(r)=2 \pi+r$. This is in fact true for $r \in\left(0, r^{*}\right]$ where $\beta^{*}$ is the unique solution of the equation $\cos \beta=\beta$, and $r^{*}=4 \cos \left(\beta^{*}\right) \approx 2.95634$. Just for sake of completeness we mention that for $r \in\left[r^{*}, 4\right]$ the optimal arc has length $L(r)=2 \pi+4 \arccos (r / 4)$. Figure 1 shows the competing $C L C$ and $C C C$-arcs. But in $\mathbb{R}^{3}$ for any $r \in(0,4]$ we find an admissible curve of length $2 \pi$, cf. Figure 2. Looking at the picture one can think of two

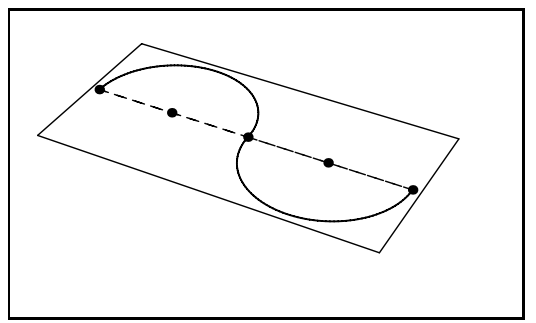

$r=4$

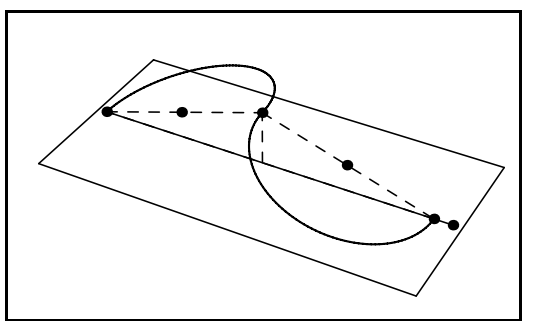

$r<4$

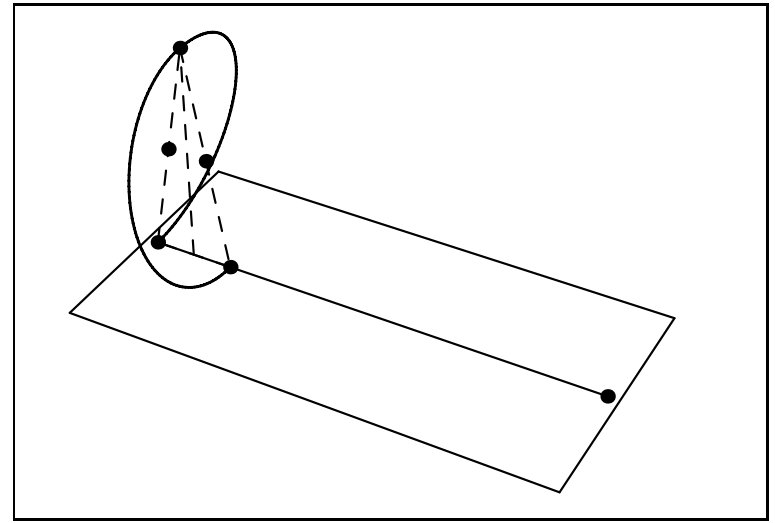

Figure 2. $r$-shifts in $\mathbb{R}^{3}$ of length $2 \pi$.

semicircles tied together but free to rotate around the common axis at the joint. We pick up the joint lifting it into the vertical z-direction while the endpoints remain in the $x y$-plane. The height of the dashed triangles is $h=\frac{1}{2} \sqrt{16-r^{2}}$. One can actually prove that these arcs are optimal, but we omit the details. At the present all we need is that the three-dimensional arcs are shorter than the best possible planar arcs.

With this example in mind it is clear that it is not trivial to prove that shortest arcs are three-dimensional, i.e. stay in a three-dimensional affine subspace compatible with the Dubins' data. Leaving the subspace 
$V\left(x_{0}, x_{1}, v_{0}, v_{1}\right)$ may decrease the length of the arc! If we think of deforming an elastic rod in the shape of a circular arc, this will add (perhaps higher-dimensional) torsion but it can reduce the curvature or, if we leave the curvature unchanged, decrease the arclength.

Nevertheless we will prove that one need not go beyond dimension 3 in the euclidian as well as the noneuclidian case.

THEOREM 1.1. In order to solve Dubins' problem in $\mathbb{R}^{n}, \mathbb{S}^{n}$ or $\mathbb{H}^{n}$, it is sufficient to solve it in $\mathbb{R}^{3}, \mathbb{S}^{3}$, resp. $\mathbb{H}^{3}$.

REMARK 1.2. In the euclidian case the previous statement may be reformulated as: every optimal arc in $\mathbb{R}^{n}$ is contained in a three-dimensional affine subspace. And if the Dubins' data $x_{0}, x_{1}, v_{0}, v_{1}$ are truly three-dimensional, i.e. if $\operatorname{det}\left(x_{1}-x_{0}, v_{0}, v_{1}\right) \neq 0$, then this subspace is unique.

In the noneuclidian case this reformulation is a little bit more sophisticated. The abstract generalization of an affine subspace would be a totally geodesic submanifold. But we rather give a concrete description. If we consider $\mathbb{S}^{n}, \mathbb{H}^{n}$ appropriately embedded into $\mathbb{R}^{n+1}$, this totally geodesic submanifold is simply the intersection of $\mathbb{S}^{n}$, resp. $\mathbb{H}^{n}$ with a subspace $\mathbb{R}^{4} \subseteq \mathbb{R}^{n+1}$. If we use the embeddings

$$
\mathbb{S}^{n}=\left\{y \in \mathbb{R}^{n+1} \mid\|y\|=1\right\}, \mathbb{H}^{n}=\left\{y \mid y_{1}^{2}-y_{2}^{2}-\cdots-y_{n+1}^{2}=1, y_{1}>0\right\},
$$

and the Dubins' data are $x_{0}, x_{1}, v_{0}, v_{1} \in \mathbb{R}^{n+1}$, then the corresponding subsphere, resp. hyperbolic subspace are given as

$$
\mathbb{S}^{n} \cap \operatorname{span}\left\{x_{0}, x_{1}, v_{0}, v_{1}\right\}, \quad \mathbb{H}^{n} \cap \operatorname{span}\left\{x_{0}, x_{1}, v_{0}, v_{1}\right\} .
$$

The reason for this simple description is that for $x_{0}, x_{1}$ in $\mathbb{S}^{n}, x_{1} \neq-x_{0}$ (not antipodal points), the geodesic through $x_{0}, x_{1}$ is actually $\mathbb{S}^{n} \cap \operatorname{span}\left\{x_{0}, x_{1}\right\}$. And a similar statement is true for $\mathbb{H}^{n}$ (cf. [16, p. 68]).

The proof of Theorem 1.1 is based on a detailed analysis of the structure of the extremals, i.e. arcs satisfying the necessary conditions provided by the (PMP). Since the local structure of extremals in dimension three has already been well analyzed, this paper has considerable overlap with the work of Monroy and Sussmann. Roughly speaking there are three types of extremals:

(1) smooth extremals with nonzero torsion,

(2a) concatenations of "circular" arcs,

(2b) concatenations of "circular" arcs and geodesic segments.

The proof of Theorem 1.1 is broken into two major steps. In Section 4 we will prove that extremals of type (1) are three-dimensional, an aspect considered neither in [18] nor in [15].

Extremals of type (2) are locally planar, they are generated by piecewise constant controls. But arbitrary concatenations of circular arcs and geodesic segments will not be three-dimensional. Therefore a detailed global analysis of the switching behavior is necessary which is carried out in sections 5 and 6 . The key result is Theorem 5.4. This is not contained in [15]. Moreover Monroy considers only $\kappa_{0}=1$ which is not important in the euclidian and spherical case, but it makes a great difference in the hyperbolic case: we will prove that the hyperbolic problem with $\kappa_{0}>1$ is in full analogy with the euclidian and spherical problem. Sussmann, on the other hand, obtains ESAIM: COCV, FEBRUARY 1998, VOL. 3, 1-22 
this theorem in [18], but only for $\mathbb{R}^{3}$. In particular the question whether an optimal arc is at most three-dimensional does not occur in that case because it is answered trivially. Since extremals of type (2b) need not be three-dimensional, our observation that an optimal arc of this type has at most three pieces and is therefore three-dimensional, is, though straightforward, crucial.

\section{DUBins' PROBLEM AS AN OPTIMAL CONTROL PROBLEM}

Sussmann considers Dubins' problem as a control problem with state space the manifold $\mathbb{R}^{n} \times \mathbb{S}^{n-1}$ (position and unit tangent vector). We use the slightly different approach of Monroy in [15] and consider it as a control problem on the Lie group $\mathrm{SE}(n)$ of euclidian motions of $\mathbb{R}^{n}$. This approach has its origin in Jurdjevic's treatment of Euler's elastica problem (cf. [3]). The Serret-Frenet equations for a curve in $\mathbb{R}^{2}$ parameterized by arclength:

$$
\begin{aligned}
& \dot{x}_{1}(s)=\cos \varphi(s), \\
& \dot{x}_{2}(s)=\sin \varphi(s), \\
& \dot{\varphi}(s)=\kappa(s),
\end{aligned}
$$

can be considered as a left-invariant control problem on the motion group $\mathrm{SE}(2)$ :

$\dot{g}(s)=g(s)\left(X_{0}+\kappa(s) X_{1}\right)$ with

$g(s)=\left(\begin{array}{ccc}1 & 0 & 0 \\ x_{1} & \cos \varphi & -\sin \varphi \\ x_{2} & \sin \varphi & \cos \varphi\end{array}\right), X_{0}=\left(\begin{array}{ccc}0 & 0 & 0 \\ 1 & 0 & 0 \\ 0 & 0 & 0\end{array}\right), X_{1}=\left(\begin{array}{ccc}0 & 0 & 0 \\ 0 & 0 & -1 \\ 0 & 1 & 0\end{array}\right)$.

This approach has two main advantages. First it easily generalizes to the noneuclidian case and allows a simultaneous treatment of the euclidian, the spherical and the hyperbolic case. Second, for invariant systems on Lie groups the (PMP) takes a very special form, so one obtains much more detailed information than for general systems, all this can be found in [3].

For dimension $n \geq 3$, we consider the group

$$
\operatorname{SE}(n)=\left\{\left(\begin{array}{cc}
1 & 0 \\
x & Q
\end{array}\right) \mid x \in \mathbb{R}^{n}, Q \in \mathrm{SO}(n)\right\}
$$

and the optimal control problem:

$$
\dot{g}=g \cdot\left(\begin{array}{ccc}
0 & 0 & 0 \\
1 & 0 & u^{T} \\
0 & -u & X
\end{array}\right), \quad X \in \mathfrak{s o}(n-1), u \in \mathbb{R}^{n-1},\|u\| \leq \kappa_{0},
$$

where the cost functional is time, i.e. we are looking for time-optimal paths steering from a given point $g_{0}$ to another point $g_{1}$.

This setting contains redundancy. One can identify a group element $g=$ $(x, Q) \in \mathrm{SE}(n)$ with a point in $\mathbb{R}^{n}$ and a positively oriented orthonormal frame attached to it. If $g(t)$ is a solution of the above ODE, then its first and second column are

$$
g(t) e_{1}=\left(\begin{array}{c}
1 \\
x(t)
\end{array}\right)
$$


and

$$
\left(\begin{array}{c}
0 \\
\dot{x}(t)
\end{array}\right)=\dot{g}(t) \epsilon_{1}=g(t)\left(\begin{array}{ccc}
0 & 0 & 0 \\
1 & 0 & u^{T} \\
0 & -u & X
\end{array}\right) e_{1}=g(t) e_{2} .
$$

So the first two columns of $g(t)$ are in one-to-one correspondence with the arc $x(t)$ and its tangent vector $v(t)=\dot{x}(t)$. The arc $x(t)$ has curvature $|\kappa(t)|=\|u(t)\|$. Monroy proves that the problems on $\mathrm{SE}(n)$ and $\mathbb{R}^{n} \times \mathbb{S}^{n-1}$ are equivalent in the sense that an optimal $g(t) \in G$ projects onto an optimal arc $x(t) \in \mathbb{R}^{n}$ and conversely every optimal $x(t)$ may be obtained this way, i.e. lifts to an optimal $g(t) \in \mathrm{SE}(n)$ (cf. [15, Theorems 2.5.1 and 2.5.2]).

This lifting is not unique for $n>2$. If $n=2$ and $v_{0}$ is a unit vector, then there is only one possible choice of another unit vector $w_{0}$ such that $\left(v_{0}, w_{0}\right)$ is a positively oriented orthonormal frame in $\mathbb{R}^{2}$. But for $n>2$ there are infinitely many possibilities.

The manifold $\mathbb{R}^{n} \times \mathbb{S}^{n-1}$ is a homogeneous space of the group $\operatorname{SE}(n)$. Let $K \subseteq \mathrm{SE}(n)$ denote the subgroup

$$
K=\left\{\left(\begin{array}{lll}
1 & 0 & 0 \\
0 & 1 & 0 \\
0 & 0 & Q
\end{array}\right) \mid Q \in \mathrm{SO}(n-1)\right\},
$$

then $\mathbb{R}^{n} \times \mathbb{S}^{n-1} \cong \mathrm{SE}(n) / K$, the canonical projection $\phi: \mathrm{SE}(n) \rightarrow \mathrm{SE}(n) / K$ is $\phi(g)=\left(g . e_{1}, g . e_{2}\right)=\left(\begin{array}{cc}1 & 0 \\ x & Q \epsilon_{1}\end{array}\right)$ sending a matrix to its first two column vectors. Let $g_{0}, g_{1} \in \mathrm{SE}(n)$ be elements projecting onto a given set of Dubins' data, i.e. $\phi\left(g_{i}\right)=\left(x_{i}, v_{i}\right)$. Then Dubins' problem is equivalent to finding a time-optimal control steering from the initial manifold $g_{0} K$ to the target manifold $g_{1} K$.

The generalization to the non-euclidian case looks similar. Let $\varepsilon \in\{-1,0$, $1\}$. Then the group $G$ one has to consider is either $\operatorname{SO}(1, n)$ or $\operatorname{SE}(n)$ or $\mathrm{SO}(n+1)$, and the ODE of the problem is

$$
\dot{g}=g \cdot\left(\begin{array}{ccc}
0 & -\varepsilon & 0 \\
1 & 0 & u^{T} \\
0 & -u & X
\end{array}\right), \quad X \in \mathfrak{s o}(n-1), u \in \mathbb{R}^{n-1},\|u\| \leq \kappa_{0} .
$$

With the same group $K \cong \mathrm{SO}(n-1)$ as above the objective is to find for given $g_{0}, g_{1} \in G$ a time-optimal arc from $g_{0} K$ to $g_{1} K$.

Lifting the problem to the group introduced redundancy which is reflected in the fact that the two-point boundary value problem is transformed into a problem with movable endpoints. As a consequence certain controls will be left completely undetermined by the (PMP). The transversality conditions will not provide any additional information, either.

Nevertheless we will eliminate these undetermined controls, i.e. we will show that one may assume that these undetermined controls vanish identically. The argument is based on symmetry considerations because the system (2.1) is not only left-invariant with respect to $G$, it is also rightinvariant with respect to $K$. This allows us to show that the optimal time to steer from $h_{0}$ to $h_{1}$ is the same for any choice of $h_{0} \in g_{0} K$ and $h_{1} \in g_{1} K$. Let $\mathfrak{g}=\mathrm{L}(G)$ denote the Lie algebra of $G$ and $\mathcal{U} \subseteq \mathfrak{g}$ the set of admissible ESAIM: COCV, FEBRUARY 1998, VOL. 3, 1-22 
control values, i.e.

$$
\mathcal{U}=\left\{\left(\begin{array}{ccc}
0 & -\varepsilon & 0 \\
1 & 0 & u^{T} \\
0 & -u & X
\end{array}\right) \mid X \in \mathfrak{s o}(n-1), u \in \mathbb{R}^{n-1},\|u\| \leq \kappa_{0}\right\} .
$$

Then $\mathcal{U}$ has the form $B+\mathfrak{k}$ where $\mathfrak{k}=\mathbf{L}(K) \cong \mathfrak{s o}(n-1)$ is the Lie algebra of $K$ and the set

$$
B=\left\{\left(\begin{array}{ccc}
0 & -\varepsilon & 0 \\
1 & 0 & u^{T} \\
0 & -u & 0
\end{array}\right) \mid u \in \mathbb{R}^{n-1},\|u\| \leq \kappa_{0}\right\}
$$

is compact and $\operatorname{Ad}(K)$-invariant because for $Q \in \mathrm{SO}(n-1)$,

$$
Y=\left(\begin{array}{ccc}
0 & -\varepsilon & 0 \\
1 & 0 & u^{T} \\
0 & -u & 0
\end{array}\right)
$$

and $k=\operatorname{diag}(1,1, Q)$ we have

$$
\operatorname{Ad}(k) Y=k Y k^{-1}=\left(\begin{array}{ccc}
0 & -\varepsilon & 0 \\
1 & 0 & (Q u)^{T} \\
0 & -Q u & 0
\end{array}\right) .
$$

LemMA 2.1. Let $u(t):[0, T] \rightarrow \mathcal{U}$ be an admissible control and $g(t)$ the corresponding trajectory. Let $k(t) \in K$ be absolutely continuous and $v(t)=$ $-k^{-1} \dot{k}$. Then $\tilde{g}(t):=g(t) k(t)$ is a trajectory of the control system (2.1), generated by the control $\tilde{u}(t)=A d\left(k^{-1}\right) u(t)-v(t)$.

Proof. We differentiate $\tilde{g}(t)$. Since $\dot{g}(t)=g(t) u(t)$ and $\dot{k}(t)=-k(t) v(t)$, we obtain

$$
\begin{aligned}
\dot{\tilde{g}}(t) & =\dot{g}(t) k(t)+g(t) \dot{k}(t)=g(t) u(t) k(t)-g(t) k(t) v(t) \\
& =g(t) k(t)\left(k^{-1} u(t) k-v(t)\right)=\tilde{g}(t)\left(\operatorname{Ad}\left(k^{-1}\right) u(t)-v(t)\right) \\
& =\tilde{g}(t) \tilde{u}(t) .
\end{aligned}
$$

Since $\operatorname{Ad}(K) \mathcal{U}=U$ and $\mathcal{U}+\mathfrak{k}=U, \tilde{u}(t) \in \mathcal{U}$ follows.

This allows to prove immediately that the minimal time is independent of a particular choice of points in $g_{0} K, g_{1} K$.

Corollary 2.2. For $g_{0}, g_{1} \in G$, let $T\left(g_{0}, g_{1}\right)$ denote the minimal time to steer from $g_{0}$ to $g_{1}$. Then, for all $k_{0}, k_{1} \in K$, we have $T\left(g_{0} k_{0}, g_{1} k_{1}\right)=$ $T\left(g_{0}, g_{1}\right)$.

Proof. Let $g(t):[0, T] \rightarrow G$ denote a time-optimal path from $g_{0}$ to $g_{1}$. Since $K \cong \mathrm{SO}(n-1)$ is connected, we can find an absolutely continuous path $k(t):[0, T] \rightarrow K$ from $k_{0}$ to $k_{1}$. Thus $\tilde{g}(t):=g(t) k(t)$ is an admissible path steering from $g_{0} k_{0}$ to $g_{1} k_{1}$ in time $T=T\left(g_{0}, g_{1}\right)$, hence $T\left(g_{0} k_{0}, g_{1} k_{1}\right) \leq$ $T\left(g_{0}, g_{1}\right)$ follows.

Applying this result to the case $g_{i}^{\prime}:=g_{i} k_{i}$ and $k_{i}^{\prime}=k_{i}^{-1}$ yields the reverse inequality. Thus equality holds.

REMARK 2.3. The same argument works, of course, with infimum instead of minimum time. Since time-optimal arcs always exist (cf. [15, Theorem 3.1.1], for example, we need not make this distinction. 
The symmetry considerations of the previous corollary and Lemma 2.1 allow to completely eliminate the redundancy introduced by lifting the system to the group $G$. This step is new and crucial. It allows us to eliminate those controls Monroy had to cope with because they are completely undetermined by the (PMP). Corollary 2.2 shows that the two-point boundary value problem and the problem with movable endpoints have the same extremals. This also explains why the transversality conditions of the (PMP) will not provide any additional information: roughly speaking the transversality conditions, if satisfied, imply that the restriction of the optimal time function $T: G \times G \rightarrow \mathbb{R}^{+}$to the submanifold $g_{0} K \times g_{1} K$ has a critical point. But this restriction is constant, so we cannot expect to obtain additional information.

\section{Application of the Maximum Principle}

As we already mentioned, for invariant control systems on Lie groups the (PMP) takes a special form providing much more detailed information than for arbitrary control systems. For a detailed discussion we refer to $[3,5,13]$. Before we apply the (PMP) we need to introduce some notation, in particular we fix a basis of $\mathfrak{g}$. Let $e_{1}, \ldots, e_{n}$ denote the standard basis of $\mathbb{R}^{n}$ and let

$$
\ell_{i}=\left(\begin{array}{cc}
0 & -\varepsilon e_{i}^{T} \\
e_{i} & 0
\end{array}\right), \text { and } L_{i j}=\left(\begin{array}{cc}
0 & 0 \\
0 & e_{i} e_{j}^{T}-e_{j} e_{i}^{T}
\end{array}\right) \text {. }
$$

With the bracket being $[X, Y]=X Y-Y X$ we obtain

$$
\begin{aligned}
{\left[\ell_{i}, \ell_{j}\right] } & =-\varepsilon L_{i j}, \\
{\left[\ell_{i}, L_{j k}\right.} & =\delta_{i j} \ell_{k}-\delta_{i k} \ell_{j}, \\
{\left[L_{i j}, L_{k l}\right] } & =\delta_{j k} L_{i l}-\delta_{i k} L_{j l}-\delta_{j l} L_{i k}+\delta_{i l} L_{j k} .
\end{aligned}
$$

(We note that Monroy uses the opposite sign, cf. [15, p. 24]). We rewrite the ODE (2.1) as

$$
\dot{g}=g\left(\ell_{1}+\sum_{i<j} u_{i j} L_{i j}\right), \quad \sum_{j=2}^{n} u_{1 j}^{2} \leq \kappa_{0}^{2} .
$$

In order to apply the (PMP) we must lift this control problem to the cotangent bundle $T^{*} G$. If we let $\mathfrak{g}^{*}$ denote the vector space dual of $\mathfrak{g}$, then one can identify $T^{*} G$ with $G \times \mathfrak{g}^{*}$. The set of smooth functions on $T^{*} G$ with the Poisson bracket $\{\cdot, \cdot\}$ is a Lie algebra. Every $X \in \mathfrak{g}$ induces a smooth function on $T^{*} G$, namely $H_{X}(g, p)=\langle p, X\rangle$. If signs for the Lie bracket $[\cdot, \cdot]$ in $\mathfrak{g}$ and the Poisson bracket $\{\cdot, \cdot\}$ are chosen properly, then the map $\left(X \mapsto H_{X}\right): \mathfrak{g} \rightarrow C^{\infty}\left(T^{*} G\right)$ is a Lie algebra homomorphism, i.e.

$$
\left\{H_{X}, H_{Y}\right\}=H_{[X, Y]} \text { for all } X, Y \in \mathfrak{g} .
$$

Now let $h_{i}, H_{i j}: G \times \mathfrak{g}^{*} \rightarrow \mathbb{R}$ denote the following functions corresponding to $\ell_{i}, L_{i j} \in \mathfrak{g}:$

$$
h_{i}(g, p)=\left\langle p, \ell_{i}\right\rangle, \quad H_{i j}(g, p)=\left\langle p, L_{i j}\right\rangle .
$$

Then the system Hamiltonian of the control problem (2.1) is

$$
\mathcal{H}_{u}(g, p)=\langle p, u\rangle=h_{1}+u_{12} H_{12}+\cdots+u_{1 n} H_{1 n}+\sum_{1<i<j}^{n} u_{i j} H_{i j}
$$


Now suppose that $g(t)$ is an optimal arc generated by the control $u(t)$. Then the special formulation of the (PMP) for invariant problems on Lie groups $([3,5,13,15])$ yields the existence of a covector $p(t) \in \mathfrak{g}^{*}$ with the following properties:

(i) $p(t)$ is not identically 0 and therefore $p(t) \neq 0$ for all $t$.

(ii) $p(t)$ satisfies a certain ODE. In implicit form, this ODE is

$$
\frac{d}{d t} \operatorname{Ad}\left(g(t)^{-1}\right)^{*} p(t) \equiv 0,
$$

so $\operatorname{Ad}\left(g(t)^{-1}\right)^{*} p(t)=: \Lambda \in \mathfrak{g}^{*}$ is constant.

(iii) The Hamiltonian is pointwise maximized by the control $u(t)$ :

$$
\mathcal{H}_{u(t)}(g(t), p(t))=\max _{v \in \mathcal{U}} \mathcal{H}_{v}(g(t), p(t)),
$$

and its value is constant, either 0 or 1 :

$$
\mathcal{H}_{u(t)}(g(t), p(t))=\lambda \in\{0,1\} .
$$

In the sequel we will call a pair $(g(t), p(t))$ an extremal if it satisfies the necessary conditions of the (PMP). We need not specify the control $u(t)$ explicitly because $\dot{g}=g u$ a.e. implies $u=g^{-1} \dot{g}$. Extremals with $\lambda=1$ are called regular or normal while extremals with $\lambda=0$ are called exceptional or abnormal.

Since there is no restriction on $u_{i j}$ with $1<i<j$, an immediate consequence of (iii) is $H_{i j}(g(t), p(t)) \equiv 0$. This is precisely the information provided by the transversality conditions. The latter imply $p(t) \perp \mathfrak{k}$ at the interval endpoints, which is equivalent to $H_{i j}(p(t))=0$ for all $1<i<j$.

As a drawback Condition (iii) does not provide any information about the controls $u_{i j}(t)$ with $1<i<j$. A symmetry argument similar to the one in Lemma 2.1 allows us to eliminate these controls.

LEMMA 3.1. Let $g(t)$ be an arbitrary trajectory of (2.1) generated by the admissible control $u$. Then there exists another trajectory $\tilde{g}$ generated by an admissible control $\tilde{u}$ with $\tilde{u}_{i j} \equiv 0$ such that $g(t) K=\tilde{g}(t) K$ for all $t$.

Proof. Since the set $\mathcal{U}$ has the form $B+\mathfrak{k}$ let us write $u(t)=u_{B}(t)+u_{\mathfrak{k}}(t)$. Then $u_{i j} \equiv 0$ for $1<i<j$ is equivalent to $u_{\mathfrak{k}}(t) \equiv 0$.

Let $k(t) \in K$ denote the solution of the ODE $\dot{k}(t)=-u_{\mathfrak{k}}(t) k(t), k(0)=1$ (identity matrix), and set $\tilde{g}(t):=g(t) k(t)$. Then we obtain

$$
\begin{aligned}
\dot{\tilde{g}}(t) & =g(t) u(t) k(t)+g(t)\left(-u_{\mathfrak{k}}(t)\right) k(t) \\
& =g(t) k(t) k(t)^{-1}\left(u(t)-u_{\mathfrak{k}}(t)\right) k(t)=\tilde{g}(t) \operatorname{Ad}\left(k(t)^{-1}\right) u_{B}(t) .
\end{aligned}
$$

Since the set $B$ is $\operatorname{Ad}(K)$-invariant, we deduce that $\tilde{u}(t):=\operatorname{Ad}\left(k(t)^{-1}\right) u_{B}(t)$ $\epsilon B$ for all $t$, or equivalently $\tilde{u}_{\mathfrak{k}} \equiv 0$. Now $\tilde{g}(t) K=g(t) k(t) K=g(t) K$, so $\tilde{g}(t)$ and $g(t)$ project onto the same arc $x(t)$.

In particular we deduce that every optimal arc can be obtained from an extremal with $u_{\mathfrak{k}} \equiv 0$. Thus we have fully eliminated the redundancy introduced by lifting the problem to the group $G$. Throughout the rest of the paper we will always assume that $(g, p)$ is an extremal generated by a control $u(t)$ with $u_{\mathfrak{k}} \equiv 0$.

Since $H_{i j} \equiv 0$ and $u_{i j} \equiv 0$ for $1<i<j$ many terms in the ODE for $p(t)$ simply disappear. Let us write $h_{i}(t), H_{1 j}(t)$ for $h_{i}(p(t)), H_{1 j}(p(t))$ by a 
slight abuse of notation. Then the derivatives $\dot{h}_{i}(t)$, can be obtained through Poisson bracketing with the system Hamiltonian $\mathcal{H}_{u}$. With our choice of sign for Lie and Poisson brackets:

$$
\dot{h}_{i}=\left\{\mathcal{H}_{u}, h_{i}\right\}=\left\{h_{1}+u_{12} H_{12}+\cdots+u_{1 n} H_{1 n}, h_{i}\right\}, \text { etc. }
$$

In particular for the functions $h_{i}, H_{1 j}$ we obtain the following brackets:

$$
\begin{aligned}
{\left[\ell_{1}, \ell_{j}\right] } & =-\varepsilon L_{1 j}, & \left\{h_{1}, h_{j}\right\} & =-\varepsilon H_{1 j}, \\
{\left[\ell_{1}, L_{1 j}\right] } & =\ell_{j}, & \left\{h_{1}, H_{1 j}\right\} & =h_{j}, \\
{\left[L_{1 j}, \ell_{j}\right] } & =\ell_{1}, & \left\{H_{1 j}, h_{j}\right\} & =h_{1}, \\
{\left[L_{1 j}, L_{1 k}\right] } & =L_{j k}, & \left\{H_{1 j}, H_{1 k}\right\} & =H_{j k} .
\end{aligned}
$$

Let us define

$$
h^{\prime}=\left(\begin{array}{c}
h_{2} \\
\vdots \\
h_{n}
\end{array}\right), H^{\prime}=\left(\begin{array}{c}
H_{12} \\
\vdots \\
H_{1 n}
\end{array}\right), \mu(t)=\left\|H^{\prime}\right\|=\sqrt{H_{12}^{2}+\cdots+H_{1 n}^{2}} .
$$

Since $H_{i j} \equiv 0$ for $i, j>1$ along an extremal, we obtain

$$
\begin{array}{rlrl}
\dot{h}_{1}=-u_{12} h_{2}-\cdots-u_{1 n} h_{n}, & & \\
\dot{h}_{2}=h_{1} u_{12}-\varepsilon H_{12}, & & \dot{H}_{12}=h_{2}, \\
& \vdots & & \vdots \\
\dot{h}_{n}=h_{1} u_{1 n}-\varepsilon H_{1 n}, & \dot{H}_{1 n}=h_{n},
\end{array}
$$

i.e.

$$
\dot{h}_{1}=-u_{B}^{T} h^{\prime}, \quad \dot{h}^{\prime}=h_{1} u_{B}-\varepsilon H^{\prime}, \quad \text { and } \quad \dot{H}^{\prime}=h^{\prime} .
$$

The function $\mu$ is the switching function. Discontinuities of the control can appear only if $\mu(t)=0$. The value of the Hamiltonian along an extremal is

$$
\mathcal{H}_{u(t)}(g(t), p(t))=h_{1}+\kappa_{0}\left\|H^{\prime}\right\|=h_{1}+\kappa_{0} \mu \equiv \lambda \in\{0,1\} .
$$

If $\mu(t)=0$, every $v \in B$ maximizes the Hamiltonian, so $u_{B}$ is completely undetermined. If $\mu(t)>0$ then the optimal control $u_{B}(t)$ is uniquely determined:

$$
u_{B}=\left(\begin{array}{c}
u_{12} \\
\vdots \\
u_{1 n}
\end{array}\right)=\frac{\kappa_{0}}{\mu} H^{\prime}
$$

Thus if $H^{\prime} \neq 0$, then $\left(h_{1}, h^{\prime}, H^{\prime}\right)$ satisfy the ODE

$$
\dot{h}_{1}=-\frac{\kappa}{\mu}\left(h^{\prime}\right)^{T} H^{\prime}, \quad \dot{h}^{\prime}=\left(\frac{\kappa h_{1}}{\mu}-\varepsilon\right) H^{\prime}, \quad \dot{H}^{\prime}=h .
$$

Besides the Hamiltonian $\mathcal{H}_{u}=h_{1}+\kappa_{0} \mu$ there are additional integrals of motion. In view of Condition (ii) of the (PMP) every function that is constant along the coadjoint orbits of $G$ in $\mathfrak{g}^{*}$ will be an integral. These functions are called Casimir functions.

ESAIM: COCV, FEBRUARY 1998, VOL. 3, 1-22 
Lemma 3.2. For each $\varepsilon \in\{-1,0,1\}$ the following two functions are Casimir functions:

$$
\begin{aligned}
J_{2}= & \sum_{i=1}^{n} h_{i}^{2}+\varepsilon \sum_{1 \leq i<j \leq n} H_{i j}^{2}, \\
J_{3}= & \sum_{1 \leq i<j<k \leq n}\left(h_{i} H_{j k}-h_{j} H_{i k}+h_{k} H_{i j}\right)^{2} \\
& +\varepsilon \sum_{1 \leq i<j<k<l \leq n}\left(H_{i j} H_{k l}-H_{j l} H_{i k}+H_{i l} H_{j k}\right)^{2} .
\end{aligned}
$$

Proof. This is proved in [15, Theorem 4.3.1] except for $J_{3}$ in the general euclidian case: $\varepsilon=0$ and $n>3$. The general euclidian case is almost contained in [2, pp. 124 et sq.] though only $n=3$ is explicitly stated. One may identify $\mathfrak{s o}(n)$ with the exterior product $\wedge^{2} \mathbb{R}^{n}$ and $\mathfrak{s e}(n)^{*}$ with $\left(\bigwedge^{2} \mathbb{R}^{n}\right) \times \mathbb{R}^{n}$. With the standard basis vectors $e_{i} \in \mathbb{R}^{n}$ and $e_{i} \wedge e_{j} \in \bigwedge^{2} \mathbb{R}^{n}$ we identify $p \in \mathfrak{s e}(n)^{*}$ with $(\beta, y)$ where

$$
\beta=\sum_{i<j} H_{i j}(p) e_{i} \wedge e_{j}, \quad \text { and } \quad y=\sum_{k} h_{k}(p) e_{k} .
$$

With these identifications and the natural actions of $\mathrm{SO}(n)$ on exterior products $\wedge^{k} \mathbb{R}^{n}$, and $\mathbb{R}^{n}$, the coadjoint action of $g=(x, Q)$ is given by (cf. [2, Eqn (19.3)]):

$$
\operatorname{Ad}(g)^{*}(\beta, y)=(Q \beta+(Q y) \wedge x, Q y)=:(\tilde{\beta}, \tilde{y})
$$

Thus $\|\tilde{\beta} \wedge \tilde{y}\|=\|(Q \beta) \wedge(Q y)+(Q y) \wedge x \wedge(Q y)\|=\|(Q \beta) \wedge(Q y)\|=\|\beta \wedge y\|$, so the map $(\beta, y) \mapsto\|\beta \wedge y\|^{2}$ is a Casimir function, and this is precisely $J_{3}$.

The functions $J_{2}, J_{3}: \mathfrak{g}^{*} \rightarrow \mathbb{R}$ will be integrals for any left-invariant control problem on $G$. In the non-euclidian case the Lie algebra $\mathfrak{g}$ is semisimple, and $J_{2}$ is actually a scalar multiple of the Cartan-Killing-form.

Since $H_{i j} \equiv 0$ for $1<i<j$ along an extremal $(g(t), p(t))$, the formulas for $J_{2}, J_{3}$ simplify significantly:

$$
\begin{aligned}
& J_{2}=h_{1}^{2}+\left\|h^{\prime}\right\|^{2}+\varepsilon\left\|H^{\prime}\right\|^{2}=h_{1}^{2}+\left\|h^{\prime}\right\|^{2}+\varepsilon \mu^{2}, \\
& J_{3}=\sum_{1<j<k \leq n} \operatorname{det}\left(\begin{array}{cc}
h_{j} & H_{1 j} \\
h_{k} & H_{1 k}
\end{array}\right)^{2} .
\end{aligned}
$$

We improve this result now:

Proposition 3.3. Along an extremal $(g, p)$ each term

$$
\operatorname{det}\left(\begin{array}{cc}
h_{j} & H_{1 j} \\
h_{k} & H_{1 k}
\end{array}\right)=h_{j} H_{1 k}-h_{k} H_{1 j}
$$

is constant.

Proof. From (3.2) we get $\dot{h}_{j}=h_{1} u_{1 j}-\varepsilon H_{1 j}$ and $\dot{H}_{1 j}=h_{j}$. Moreover the equality $H^{\prime}=\mu \kappa_{0}^{-1} u$ holds, regardless whether $\mu>0$ or $\mu=0$. Hence we 
obtain

$$
\begin{aligned}
& \frac{d}{d t}\left(h_{j} H_{1 k}-h_{k} H_{1 j}\right)=\dot{h}_{j} H_{1 k}+h_{j} \dot{H}_{1 k}-\dot{h}_{k} H_{1 j}-h_{k} \dot{H}_{1 j} \\
& \quad=\left(h_{1} u_{1 j}-\varepsilon H_{1 j}\right) H_{1 k}+h_{j} h_{k}-\left(h_{1} u_{1 k}-\varepsilon H_{1 k}\right) H_{1 j}-h_{k} h_{j} \\
& \quad=h_{1}\left(u_{1 j} H_{1 k}-u_{1 k} H_{1 j}\right)=h_{1} \operatorname{det}\left(\begin{array}{cc}
u_{1 j} & H_{1 j} \\
u_{1 k} & H_{1 k}
\end{array}\right) \\
& \quad=\frac{h_{1} \mu}{\kappa_{0}} \operatorname{det}\left(\begin{array}{cc}
u_{1 j} & u_{1 j} \\
u_{1 k} & u_{1 k}
\end{array}\right) \equiv 0 .
\end{aligned}
$$

Sussmann observed that for a geometrically satisfying treatment one has to consider the exterior product $\wedge \mathbb{R}^{n}$. This is in accordance with the description of the coadjoint action. The previous proposition implies that $h^{\prime} \wedge H^{\prime} \in \wedge^{2} \mathbb{R}^{n}$ is a constant of motion, in fact $J_{3}=\left\|e_{1} \wedge H^{\prime} \wedge h^{\prime}\right\|^{2}$. In dimension 3 everything could be expressed in terms of the vector product because the latter provides an isomorphism between $\bigwedge^{2} \mathbb{R}^{3}$ and $\mathbb{R}^{3}$.

\section{Smooth Extremals With TORSion}

In this section we only consider extremals with $J_{3}>0$. We will prove that they are smooth and three-dimensional.

Lemma 4.1. Let $(g, p)$ be an extremal with $J_{3}>0$. Then $\mu(t)>0$ for all $t \in \mathbb{R}$ and $g(t), p(t), u(t)$ are analytic.

Proof. If $\mu\left(t_{0}\right)=0$ for some $t_{0} \in \mathbb{R}$, then $H^{\prime}\left(t_{0}\right)=0$, whence $J_{3}\left(p\left(t_{0}\right)\right)=0$. Since $J_{3}$ is constant along $p(t)$, this implies $J_{3} \equiv 0$. Since we assumed $J_{3}>0$, this is impossible, hence $\mu(t)>0$ for all $t$ follows.

Since the righthand side of the ODE (3.2) is well-defined and analytic whenever $\mu>0, p(t)$ must be analytic. As $\mu(t)>0$ yields $u_{B}(t)=$ $\kappa_{0} \mu(t)^{-1} H^{\prime}(t)$, the control $u$ is analytic as well as $g(t)$.

The ODE (3.2) shows that all higher order derivatives of $h^{\prime}, H^{\prime}$ are linear combinations of $h^{\prime}, H^{\prime}$. Thus we obtain:

Corollary 4.2. The vectors $h^{\prime}(t), H^{\prime}(t) \in \mathbb{R}^{n-1}$ always stay in a fixed, at most two-dimensional subspace $V_{0} \subseteq \mathbb{R}^{n-1}$.

Proof. Take $t_{0} \in \mathbb{R}$ arbitrary but fixed and let $V_{0}=\mathbb{R} h^{\prime}\left(t_{0}\right)+\mathbb{R} H^{\prime}\left(t_{0}\right) \subseteq$ $\mathbb{R}^{n-1}$. Take $v_{0} \in V_{0}^{\perp}$ arbitrary but fixed. Since $h^{\prime}, H^{\prime}$ are analytic, the functions

$$
f(t)=\left\langle v_{0}, h^{\prime}(t)\right\rangle, \quad g(t)=\left\langle v_{0}, H^{\prime}(t)\right\rangle
$$

are analytic, too. In view of the ODE (3.2) all derivatives of $h^{\prime}, H^{\prime}$ at $t_{0}$ lie in $V_{0}$. Since $v_{0}$ is perpendicular to $V_{0}$, all derivatives of $f$ and $g$ at $t_{0}$ must vanish. Since $f, g$ are analytic, they must vanish identically. Thus $h^{\prime}(t), H^{\prime}(t) \subseteq v_{0}^{\perp}$. Since $v_{0} \in V_{0}^{\perp}$ was arbitrary, we deduce $h^{\prime}(t), H^{\prime}(t) \in V_{0}$ for all $t$.

Since the control $u(t)$ is given by $u_{B}=\kappa_{0} \mu^{-1} H^{\prime}$, this implies that $u_{B}(t)$ lies in a two-dimensional subspace of $\mathbb{R}^{n-1}$.

LEMMA 4.3. We may assume without loss of generality that $u_{B}=\left(u_{12}, u_{13}\right.$, $0, \ldots, 0)$. 
Proof. Since $u_{B}(t)$ is contained in a fixed two-dimensional subspace $V_{0} \subseteq$ $\mathbb{R}^{n-1}$, we can find a $Q \in \mathrm{SO}(n-1)$ such that $Q V_{0}=\mathbb{R}^{2} \times\{0\} \subseteq \mathbb{R}^{n-1}$. We have

$$
\dot{g}=g X_{u_{B}}, \quad \text { with } \quad X_{u_{B}}=\left(\begin{array}{ccc}
0 & -\varepsilon & 0 \\
1 & 0 & u_{B}^{T} \\
0 & -u_{B} & 0
\end{array}\right) .
$$

Let $k=\operatorname{diag}\left(1,1, Q^{T}\right) \cong \mathrm{SO}(n-1) \cong K$ and set $\tilde{g}:=g k$. Then by Lemma $2.1 \tilde{g}$ projects onto the same curve as $g$, and $\dot{\tilde{g}}=\tilde{g} \operatorname{Ad}\left(k^{-1}\right) X_{u_{B}}$. Since $\operatorname{Ad}\left(k^{-1}\right) X_{u_{B}}=X_{Q u_{B}}$ and $Q u_{B} \in \mathbb{R}^{2} \times\{0\} \subseteq \mathbb{R}^{n-1}$, our claim follows.

Thus we obtain:

THEOREM 4.4. If $(g, p)$ is an extremal with $J_{3}>0$, then the arc $x(t)=$ $g(t) e_{1}$ is three-dimensional.

Proof. By the previous lemma we may assume w.l.o.g. that the controls $u_{14}, \ldots, u_{1 n}$ vanish identically. Thus $u(t) \in \operatorname{span}\left\{\ell_{1}, L_{12}, L_{13}\right\}$. Let $\mathfrak{g}_{3}$ denote either $\mathfrak{s e}(3), \mathfrak{s o}(1,3)$, or $\mathfrak{s o}(4)$, i.e. the Lie algebra one has to consider for the three-dimensional Dubins' problem sitting in the upper 4 by 4 -block of $\mathfrak{g}$, and let $G_{3}$ denote the corresponding subgroup of $G$. Since the Lie algebra generated by $\left\{\ell_{1}, L_{12}, L_{13}\right\}$ is $\mathfrak{g}_{3}$, we deduce that $g(t) \in g(0) G_{3}$. Thus $x(t)$ is a solution of the three-dimensional problem shifted by $g(0) \in G$ into a different position in $\mathbb{R}^{n}, \mathbb{H}^{n}$, resp. $\mathbb{S}^{n}$.

The extremals with $J_{3}>0$ correspond to smooth three-dimensional arcs with constant curvature $\kappa_{0}$ and nonvanishing torsion $\tau$. For dimension three Monroy has actually proved [15, Theorem 6.2.1]:

Theorem 4.5 (Monroy's Torsion Formula). Let $(g, p)$ be an extremal with $J_{3}>0$. Then the corresponding arc has constant curvature $\kappa_{0}$ and its torsion is

$$
\tau(t)=-\frac{\sqrt{J_{3}}}{\mu(t)^{2}}
$$

where again $\mu(t)=\left\|H^{\prime}(t)\right\|$ is the switching function.

Due to our reduction, the same formula follows for higher dimensions, too. For more information on these arcs we refer to [18] and [15]. Sussmann claims in [18] that these arcs are actually strict local minimizers and that the torsion cannot be arbitrarily large. In $\mathbb{R}^{3}$ with $\kappa_{0}=1$ it is not hard to show that $\mu \equiv \mu^{*}>0$ is possible. In such a case the torsion is constant too, $\tau \equiv \tau^{*}<0$. Further analysis shows that only $\tau^{*} \in[-1,0)$ is possible. In particular $\tau^{*}=-1$ is achieved if and only if $\lambda=0$ and $J_{2}^{2}=4 J_{3}>0$. This seems to be noteworthy because it shows that in higher dimensions the abnormal extremals yield arcs that cannot be obtained (not even locally) from regular extremals.

Monroy solves the ODE for $p$ in the three-dimensional case by quadratures. For the euclidian case $\mathbb{R}^{3}$ he also derives formulas expressing the arc $x(t)$ in terms of the switching function $\mu$ and $\int \mu(t) d t$. He also derives an ODE for $\mu$, thus proving that $\mu$ may be expressed in terms of elliptic functions. We will make use of this ODE, too, see Eqn. (6.1). 
Finally we add the new observation that again the hyperbolic problem has an extraordinary property. If we consider $\varepsilon=-1, \kappa_{0}=1$ and $J_{3}>0$, $\lambda=0$, then the $\operatorname{ODE}(3.2)$ yields $\dot{h}^{\prime}=0$, hence $h^{\prime}(t)$ is constant, nonzero (because of $J_{3}>0$ ), and $H^{\prime}(t)=a+t b$ with constant, linear independent vectors $a, b \in \mathbb{R}^{n-2}$, and $h_{1}=\mu=\left\|H^{\prime}\right\|$. In this case it is even possible to integrate the ODE for $g(t)$ in terms of elementary functions, see [10].

\section{Extremals Without TORsion: The SWitching PATtern}

The function $\mu(t)$ is called the switching function because its zeros are linked to discontinuities of the controls: switches can occur only if $\mu=0$. Geometrically the case $J_{3}=0$ corresponds to the planar Dubins' problem, it yields the arcs following Dubins' pattern.

LEMMA 5.1. If $(g, p)$ is an extremal with $J_{3}=0$ and $I$ is an interval where $\mu(t)>0$, then $u(t)$ is constant in $I$.

Proof. Since $\mu>0$ in $I$ we have $u=\mu^{-1} \kappa_{0} H^{\prime}$. At least one component of $H^{\prime}$ does not vanish identically. We simply differentiate

$$
\frac{d}{d t} \frac{H_{1 j}}{H_{1 k}}=\frac{\dot{H}_{1 j} H_{1 k}-H_{1 j} \dot{H}_{1 k}}{H_{1 k}^{2}}=\frac{h_{j} H_{1 k}-H_{1 j} h_{k}}{H_{1 k}^{2}}=\frac{1}{H_{1 k}^{2}} \operatorname{det}\left(\begin{array}{cc}
h_{j} & H_{1 j} \\
h_{k} & H_{1 k}
\end{array}\right) \text {. }
$$

The determinant vanishes because of $J_{3}=0$, so $H_{1 j}=c_{j k} H_{1 k}$ with a constant $c_{j k}$. Hence $H^{\prime}(t)=H_{1 k}(t)\left(c_{2 k}, c_{3 k}, \ldots, c_{n, k}\right)^{T}$ and $u(t)=\kappa_{0} \frac{1}{\left\|H^{\prime}\right\|} H^{\prime}$ is constant in $I$. Equivalently we can write $H^{\prime}(t)=\mu(t) c_{I}$ with a constant vector $c_{I}$ of length $\left\|c_{I}\right\|=1$, and $u_{B} \equiv \kappa_{0} c_{I}$.

The corresponding arc will be a circular arc. Geodesic arcs appear only in the following situation (cf. [15, Propositions 4.6.1 and 4.6.2]).

LEMMA 5.2. If $(g, p)$ is an extremal such that $\mu(t) \equiv 0$ in an open interval I, then $J_{3}=0, J_{2}=1, \lambda=1$ and in $I$ we have

$$
h_{1} \equiv 1, \quad h^{\prime} \equiv 0, \quad H^{\prime} \equiv 0, \quad \text { and } \quad u \equiv 0 .
$$

Proof. If $\mu \equiv 0$ in $I$, then $H^{\prime} \equiv 0$ because $\left\|H^{\prime}\right\|=\mu$. Thus $0 \equiv \dot{H}^{\prime}=h^{\prime}$ yields $h^{\prime} \equiv 0$. Since $p(t) \neq 0$ by the (PMP), $h_{1} \neq 0$ follows. Therefore $0 \equiv \dot{h}^{\prime}=h_{1} u$ yields $u \equiv 0$. Finally $h_{1}+\kappa_{0} \mu-\lambda \equiv 0$ yields $h_{1} \equiv \lambda \in\{0,1\}$. As $h_{1} \neq 0, h_{1}=\lambda=1$ follows. Evaluating $J_{2}, J_{3}$ at $\left(h_{1}, h^{\prime}, H^{\prime}\right)=(1,0,0)$ yields $J_{3}=0$ and $J_{2}=1$.

Thus we obtain:

Corollary 5.3. If $(g, p)$ is an extremal with $J_{3}=0$, then the corresponding arc is a concatenation of circular arcs and geodesic segments.

So locally these arcs are even planar. Monroy obtains the previous corollary from his torsion formula. But he does not determine the precise switching patterns. Since arbitrary concatenations of circular arcs will not be planar, we must determine the global switching behavior of optimal controls. Besides Monroy only considers $\kappa_{0}=1$. This does not affect the structure of optimal arcs in $\mathbb{R}^{n}$ and $\mathbb{S}^{n}$, but for $\mathbb{H}^{n}$ it makes a great difference. Since we also want to cover that case, we will have to adjust and refine some of his arguments. Since this requires a detailed analysis of the behavior of ESAIM: COCV, FEBRUARY 1998, VOL. 3, 1-22 
the switching function $\mu$, we state our final result first. It generalizes Sussmann's three-dimensional euclidian results (cf. [18, Theorem 1]) to higher dimensions as well as to the non-euclidian manifolds $\mathbb{S}^{n}$ and $\mathbb{H}^{n}$.

Theorem 5.4. Suppose that $\kappa_{0}^{2}+\varepsilon>0$. Let $(g, p)$ be an extremal with $J_{3}=0$. Then the optimal control follows one of the following patterns:

(i) The control is $u(t) \equiv u_{0}$ is constant, $\left\|u_{0}\right\|=\kappa_{0}$. The corresponding arc is a circle and therefore genuinely planar.

(ii) For a fixed $u_{0}$ with $\left\|u_{0}\right\|=\kappa_{0}$ the control $u(t)$ follows the pattern

$$
u_{0} \vdash-u_{0} \vdash u_{0} \vdash-u_{0} \vdash \ldots
$$

and the time $T^{*}$ between successive switches is always the same, between a half period and a full period:

$$
T^{*} \in\left[\frac{\pi}{\sqrt{\kappa_{0}^{2}+\varepsilon}}, \frac{2 \pi}{\sqrt{\kappa_{0}^{2}+\varepsilon}}\right) .
$$

The corresponding arc $x(t)$ is a concatenation of circular arcs $C \ldots C$, all intermediate circles have the same length, they are at least semicircles, but shorter than a full circle, and all these arcs are in the same 'plane.' So the arc $x(t)$ is genuinely planar.

(iii) For arbitrary but fixed $u_{0}, u_{1}, u_{2} \ldots$ with $\left\|u_{i}\right\|=\kappa_{0}$ the control $u(t)$ follows the pattern

$$
u_{0} \vdash 0 \vdash u_{1} \vdash 0 \vdash u_{2} \vdash 0 \vdash u_{3} \ldots
$$

The intervals where $u(t)=0$ may have arbitrary nonnegative length. The length of an interval between two switches where $u(t) \equiv u_{i}$, is an integer multiple of the full period $2 \pi / \sqrt{\kappa_{0}^{2}+\varepsilon}$. The corresponding arc is a concatenation of circles and geodesics $C L C L C \ldots$, and a $C$ between two $L$ 's must be a full circle. Hence an optimal arc of this type has at most one $L$, so it must be CLC. Therefore it is at most three-dimensional

REMARK 5.5. Even though extremals of types (ii) and (iii) may have infinitely many switchings, a clustering sequence of switching points (Fuller's phenomenon, see [8]) cannot appear because every interval where $\mu>0$ has length at least $\pi / \sqrt{\kappa_{0}^{2}+\varepsilon}$.

But optimal arcs will actually have at most two switches. For $\mathbb{R}^{2}$ this is the major result in Dubins' original paper. For optimal arcs of type (iii) we already observed this to be true as a consequence of the (PMP). For arcs of type (ii) this is another theorem. The euclidian case follows from Dubins' result because arcs of type (ii) are automatically planar. The spherical case was solved by Monroy [15, Corollary 5.4.2], the hyperbolic case is solved in $[12]$.

If $\kappa_{0}^{2}+\varepsilon \leq 0$, the switching patterns are similar but much simpler:

Theorem 5.6. Suppose that $\kappa_{0}^{2}+\varepsilon \leq 0$. Let $(g, p)$ be an extremal with $J_{3}=0$. Then the optimal control $u(t)$ follows one of the following patterns:

(i) The control is $u(t) \equiv u_{0}$ is constant, $\left\|u_{0}\right\|=\kappa_{0}$. The corresponding arc is a circular arc $C$ and therefore genuinely planar. 
(ii) There is exactly one switch. For a fixed $u_{0}$ with $\left\|u_{0}\right\|=\kappa_{0}$ there is $t_{0} \in \mathbb{R}$ such that the control is

$$
u(t)= \begin{cases}u_{0}, & \text { if } t \in\left(-\infty, t_{0}\right) \\ -u_{0}, & \text { if } t \in\left(t_{0}, \infty\right)\end{cases}
$$

The corresponding arc $x(t)$ is a concatenation of two circular arcs $C C$ lying in the same 'plane'. So the arc $x(t)$ is genuinely planar.

(iii) There are two switches at $t_{1} \leq t_{2}$. For arbitrary but fixed $u_{1}, u_{2}$ with $\left\|u_{i}\right\|=\kappa_{0}$ the control is

$$
u(t)= \begin{cases}u_{1}, & \text { if } t \in\left(-\infty, t_{1}\right) \\ 0, & \text { if } t \in\left(t_{1}, t_{2}\right) \\ u_{2}, & \text { if } t \in\left(t_{2}, \infty\right)\end{cases}
$$

The corresponding arc is of the form CLC and therefore it is at most three-dimensional.

REMARK 5.7. That a control following the alternating pattern $u_{0} \vdash-u_{0} \ldots$ yields a 'planar' arc follows from the fact that the subalgebra generated by $\left\{\ell_{1}, X_{u_{0}}\right\}$ is isomorphic to $\mathfrak{s e}(2), \mathfrak{s o}(1,2)$, resp. $\mathfrak{s o}(3)$. So the same argument as in the proof of Theorem 4.4 shows that $x(t)$ is a planar curve.

The geometric meaning of the condition $\kappa_{0}^{2}+\varepsilon>0$, resp. $\leq 0$ is quickly explained. Let

$$
X_{u}=\left(\begin{array}{ccc}
0 & -\varepsilon & 0 \\
1 & 0 & -u^{T} \\
0 & u & 0
\end{array}\right), \quad \text { with }\|u\|=\kappa_{0} .
$$

Then $X_{u}^{3}=-\left(\kappa_{0}^{2}+\varepsilon\right) X_{u}$. If $\kappa_{0}^{2}+\varepsilon>0$, then $\exp \left(\mathbb{R} X_{u}\right)$ is a circle group. With $w:=\sqrt{\kappa_{0}^{2}+\varepsilon}$ we obtain:

$$
\exp \left(t X_{u}\right)=I+\frac{\sin (w t)}{w} X_{u}+\frac{1-\cos (w t)}{w^{2}} X_{u}^{2} .
$$

And the period is $T^{*}=\frac{2 \pi}{w}$, i.e. $\exp \left(T^{*} X_{u}\right)=I$. The $\operatorname{arc} \exp \left(t X_{u}\right) e_{1}$ is a circular arc. And an arc over the full period $T^{*}$ is a full circle.

In the euclidian and spherical case, we always have $\kappa_{0}^{2}+\varepsilon>0$. In the hyperbolic case $0<\kappa_{0}^{2}+\varepsilon=\kappa_{0}^{2}-1$ is equivalent to $\kappa_{0}>1$. This is the controllable hyperbolic case (cf. [11]).

In the noncontrollable hyperbolic case, i.e. $\varepsilon=-1$ and $\kappa_{0} \leq 1$, we have $X_{u}^{3}=\left(1-\kappa_{0}^{2}\right) X_{u}$ and therefore with $w:=\sqrt{1-\kappa_{0}^{2}}$ we obtain:

$$
\begin{array}{ll}
\exp \left(t X_{u}\right)=I+t X_{u}+\frac{t^{2}}{2} X_{u}^{2}, & \text { if } \kappa_{0}=1, \\
\exp \left(t X_{u}\right)=I+\frac{\sinh (w t)}{w} X_{u}+\frac{\cosh (w t)-1}{w^{2}} X_{u}^{2}, & \text { if } \kappa_{0}<1 .
\end{array}
$$

So in this case the 'circular arcs' are never closed. Thinking of traveling in a car at constant speed this means it is impossible to return to the starting point. It was proved in [11] that in this case Dubins' problem in $\mathbb{H}^{n}$ is not controllable, i.e. there are boundary conditions $x_{0}, v_{0}, x_{1}, v_{1}$ for which it is impossible to find an admissible arc. 


\section{ANALYZING THE SWITCHING BEHAVIOR}

In order to obtain this information about the extremals we must take a closer look at the switching function. Mon roy has proved that the switching function $\mu$ satisfies a certain ODE, see [15, Theorem 4.5.1]. Since we consider $\kappa_{0}>0$ rather than just $\kappa_{0}=1$, we must modify his proof slightly (p. 39, first line, we have $h_{1}=\lambda-\kappa_{0} \mu$ rather than $h_{1}=\lambda-\mu$ ) and obtain

$$
(\mu \dot{\mu})^{2}=-\left(\kappa_{0}^{2}+\varepsilon\right) \mu^{4}+2 \kappa_{0} \lambda \mu^{3}+\left(J_{2}-\lambda^{2}\right) \mu^{2}-J_{3}=: f(\mu) .
$$

For $J_{3}=0$, this ODE simplifies to

$$
\mu^{2}(\dot{\mu})^{2}=\mu^{2}\left(-\left(\kappa_{0}^{2}+\varepsilon\right) \mu^{2}+2 \kappa_{0} \lambda \mu+J_{2}-\lambda^{2}\right)=\mu^{2} F(\mu) .
$$

If $\mu>0$ in an interval $I$, then either $\dot{\mu}=+\sqrt{F(\mu)}$ or $\dot{\mu}=-\sqrt{F(\mu)}$ because $\mu$ is smooth in $I$. Since $F(\mu)$ is a quadratic polynomial, the ODE is integrable in terms of elementary functions (cf. [15, Corollary 4.5.1]). We omit integration of this ODE. We note that $\mu$ is defined for all $t$. For the control is locally constant, thus $g(t)$ is just a product of exponentials. So $(g(t), p(t))$ are defined for all $t$, hence so is $\mu$.

One consequence of this ODE is that $\mu$ cannot assume arbitrary values. The righthand side $\mu^{2} F(\mu)$ has to be nonnegative. Therefore the possible range of $\mu$ is

$$
\mathcal{R}=\left\{\mu \mid \mu \geq 0, \mu^{2} F(\mu) \geq 0\right\}=\{0\} \cup\left(F^{-1}\left(\mathbb{R}^{+}\right) \cap(0, \infty)\right) .
$$

The case $F(0)=J_{2}-\lambda^{2}<0$ is the simplest case.

LEMMA 6.1. If $(g, p)$ is an extremal with $J_{3}=0$ and $J_{2}-\lambda^{2}<0$, then $\mu(t)>0$ for all $t$, the corresponding control $u(t)$ is a constant $u_{0}$ with $\left\|u_{0}\right\|=\kappa_{0}$, and the corresponding arc is a circular arc.

Proof. If $F(0)<0$, then $\mu^{2} F(\mu)<0$ for $\mu>0$ sufficiently small. Since $\mu$ is continuous, either $\mu(t)>0$ for all $t$ or $\mu(t) \equiv 0$ follows. If $\mu \equiv 0$, then Lemma 5.2 yields $J_{2}=1$ and $\lambda=1$, hence $F(0)=0$, a contradiction, so this is impossible. Thus $\mu(t)$ never vanishes, the control $u(t)$ is constant, and the corresponding arc $x(t)$ is a circular arc.

Next we consider what kind of information we get about switches. We claim that if $F(0)>0$, then we can only have an alternating switching pattern, i.e. switches of the form $u_{0} \vdash-u_{0}$.

LemMa 6.2. Let $(g, p)$ be an extremal with $J_{3}=0$ and $J_{2}-\lambda^{2}>0$. Let $t_{l}<t^{*}<t_{r}$ be points such that $\mu\left(t^{*}\right)=0$ and $\mu(t)>0$ in $\left(t_{l}, t^{*}\right) \cup\left(t^{*}, t_{r}\right)$. Let $u_{l}, u_{r}$ denote the values of the control in these intervals. Then

$$
u_{r}=-u_{l} \text {. }
$$

Proof. Let $I_{l}, I_{r}$ denote the two intervals. Since $\mu>0$ in each of these, either $\dot{\mu}=+\sqrt{F(\mu)}$ or $\dot{\mu}=-\sqrt{F(\mu)}$. Thus $\mu$ is either increasing or decreasing. As $\mu\left(t^{*}\right)=0$, it must be decreasing in $I_{l}$ and increasing in $I_{r}$, hence

$$
\dot{\mu}(t)= \begin{cases}-\sqrt{F(\mu(t))}, & \text { in } I_{l}, \\ +\sqrt{F(\mu(t))}, & \text { in } I_{r} .\end{cases}
$$

Since $\mu$ is smooth in $I_{l}, I_{r}$ we look at the one-sided limits of $\dot{\mu}$ :

$$
\lim _{t \rightarrow t^{*}-} \dot{\mu}(t)=-\sqrt{F(0)}<0, \text { and } \lim _{t \rightarrow t^{*}+} \dot{\mu}(t)=\sqrt{F(0)}>0 .
$$


Now we recall that in $I_{l}, I_{r}$ we have $\mu(t) u=\kappa_{0} H^{\prime}(t)$ and therefore $\dot{\mu} u=$ $\kappa_{0} \dot{H}^{\prime}=\kappa_{0} h^{\prime}$. Now $h^{\prime}$ is continuous. So we compute the one-sided limits at $t^{*}$ and get

$$
-\sqrt{F(0)} u_{l}=\lim _{t \rightarrow t^{*}-} \dot{\mu}(t) u_{l}=\kappa_{0} h^{\prime}\left(t^{*}\right)=\lim _{t \rightarrow t^{*}+} \dot{\mu}(t) u_{r}=\sqrt{F(0)} u_{r} .
$$

Since $F(0)>0$, this proves $u_{r}=-u_{l}$.

We notice that if $F(0)=0$, then we do not obtain any information about the values of the control, besides being constant in $I_{l}, I_{r}$. So our final task is to determine the length of an interval where $\mu(t)>0$. This also makes sure that there cannot be clusters of isolated zeros of $\mu$ (cf. [15, Proposition 4.6.3]).

We distinguish between the two cases $\kappa_{0}^{2}+\varepsilon>0$ and $\kappa_{0}^{2}+\varepsilon \leq 0$.

Lemma 6.3. Suppose that $\kappa_{0}^{2}+\varepsilon \leq 0$. Let $(g, p)$ be an extremal with $J_{3}=0$ and $F(0) \geq 0$. If $F(0)>0$, then there is at most one alternating switch. If $F(0)=0$, the control has the form $u_{1} \vdash 0 \vdash u_{2}$.

Proof. We do not need to integrate the ODE (6.2). It suffices to analyze the behavior of its flow. Since $\kappa_{0}^{2}+\varepsilon \leq 0$, the graph of $F(\mu)$ is a parabola open to the top or a line. Since $F^{\prime}(0)=2 \lambda \kappa_{0} \geq 0$, we obtain $\mathcal{R}=[0, \infty)$ and $F(\mu)>0$ for $\mu \in(0, \infty)$.

Now let $t_{0}$ be a point with $\mu\left(t_{0}\right)>0$. Let $I$ denote the largest interval containing $t_{0}$ where $\mu(t)>0$. Then we have two possibilities.

If $\dot{\mu}\left(t_{0}\right)>0$, then $\dot{\mu}=\sqrt{F(\mu)}>0$ in $I$, thus $\mu$ is increasing in $I$ and therefore $\mu(t)>0$ for all $t>t_{0}$ follows. Hence there are no switches after $t_{0}$.

If $\dot{\mu}\left(t_{0}\right)<0$, then $\dot{\mu}=-\sqrt{F(\mu)}$ in $I$, so $\mu$ will decrease until it reaches 0 , say at $t^{*}$ and $\mu(t)>0$ for all $t<t^{*}$. Thus there are no switches before $t^{*}$.

Hence we obtain that if $\mu\left(t_{0}\right)>0$, then $t_{0}$ may lie either before or after a switch, but never between two switches. In other words, if $t_{1}<t_{2}$ are zeros of $\mu$, then $\mu \equiv 0$ in $\left[t_{1}, t_{2}\right]$. By Lemma 5.2 this can happen only if $J_{2}=1$, $\lambda=1$, i.e. $F(0)=0$.

So if $F(0)>0$, then $\mu$ has at most one zero $t_{1}$. Now Lemma 6.2 applies proving that at $t_{1}$ there is an alternating switch: $u_{0} \vdash-u_{0}$

If $F(0)=0$, then let $t_{1}=\inf \{t \mid \mu(t)=0\}$ and $t_{2}=\sup \{t \mid \mu(t)=0\}$. Then $\mu \equiv 0$ in $\left[t_{1}, t_{2}\right]$ follows as well as $\mu>0$ in $\left(-\infty, t_{1}\right) \cup\left(t_{2}, \infty\right)$. So the corresponding control is

$$
\begin{cases}u_{1}, & \text { in }\left(-\infty, t_{1}\right), \\ 0, & \text { in }\left(t_{1}, t_{2}\right), \\ u_{2}, & \text { in }\left(t_{2}, \infty\right) .\end{cases}
$$

This finishes the proof of Theorem 5.6.

Finally let us consider the case $\kappa_{0}^{2}+\varepsilon>0$ and $F(0) \geq 0$. We must compute the length of a maximal interval where $\mu>0$. Since $F$ is a quadratic function with negative leading coefficient, and $F(0) \geq 0$, it factorizes as

$$
F(\mu)=\left(\kappa_{0}^{2}+\varepsilon\right)(\mu-a)(b-\mu), \quad \text { with } a \leq 0 \leq b .
$$


We observe that

$$
\int \frac{d \mu}{\sqrt{(\mu-a)(b-\mu)}}=\arcsin \left(\frac{\mu-\frac{b+a}{2}}{\frac{b-a}{2}}\right) .
$$

Of course we could integrate the ODE for $\mu$, but actually all we need is the time between switches, i.e. the maximal length of an interval $I$ such that $\mu(t)>0$ in $I$.

Lemma 6.4. Suppose that $\kappa_{0}^{2}+\varepsilon>0$ and let $(g, p)$ be an extremal with $J_{3}=0$ and $J_{2}-\lambda^{2} \geq 0$. Then every maximal interval $I$ where $\mu(t)>0$ has length

$$
\ell(I) \in\left[\frac{\pi}{\sqrt{\kappa_{0}^{2}+\varepsilon}}, \frac{2 \pi}{\sqrt{\kappa_{0}^{2}+\varepsilon}}\right] .
$$

More precisely $\ell(I)=2 \pi / \sqrt{\kappa_{0}^{2}+\varepsilon}$ if and only if $J_{2}-\lambda^{2}=0$, and $\ell(I)=$ $\pi / \sqrt{\kappa_{0}^{2}+\varepsilon}$ if and only if $\lambda=0$.

Proof. Since $\mu(t)>0 \in I$ and $\mathcal{R}=[0, b], b>0$ follows, and $\mu(t) \in(0, b]$ for $t \in I$. Let $t_{0} \in I$ be such that $\mu\left(t_{0}\right)=b$. Then in $I \mu$ must be increasing for $t<t_{0}$ and decreasing for $t>t_{0}$. The time to decrease from $b$ to 0 is

$$
T^{*}=\int_{b}^{0} \frac{d \mu}{-\sqrt{F(\mu)}}=\int_{0}^{b} \frac{d \mu}{\sqrt{F(\mu)}}=\frac{1}{\sqrt{\left(\kappa_{0}^{2}+\varepsilon\right)}} \int_{0}^{b} \frac{d \mu}{\sqrt{(\mu-a)(b-\mu)}} .
$$

Thus the length of $I$ is

$$
\begin{aligned}
\ell(I) & =2 T^{*}=\left.\frac{2}{\sqrt{\kappa_{0}^{2}+\varepsilon}} \arcsin \left(\frac{\mu-\frac{b+a}{2}}{\frac{b-a}{2}}\right)\right|_{0} ^{b} \\
& =\frac{2}{\sqrt{\kappa_{0}^{2}+\varepsilon}}\left(\frac{\pi}{2}+\arcsin \frac{b+a}{b-a}\right) .
\end{aligned}
$$

From $F(\mu)=\left(\kappa_{0}^{2}+\varepsilon\right)(\mu-a)(b-\mu)=\left(\kappa_{0}^{2}+\varepsilon\right)\left(-\mu^{2}+(a+b) \mu-a b\right)$ we obtain $a+b=\frac{2 \lambda \kappa_{0}}{\kappa_{0}^{2}+\varepsilon} \geq 0$. Thus

$$
\ell(I) \in\left[\frac{\pi}{\sqrt{\kappa_{0}^{2}+\varepsilon}}, \frac{2 \pi}{\sqrt{\kappa_{0}^{2}+\varepsilon}}\right] .
$$

The left endpoint is obtained if and only if $a+b=0$. Since $\kappa_{0}>0$, this happens if and only if $\lambda=0$. The right endpoint is attained when $a=0$. This is equivalent to $0=F(0)=J_{2}-\lambda^{2}$.

In particular we obtain that the zeroes of $\mu$ are essentially isolated (cf. [15, Proposition 4.6.3]).

Corollary 6.5. If $(g, p)$ is an extremal with $J_{3}=0$ and $J_{2}-\lambda^{2}>0$, then the corresponding control is alternating and the time between successive switches is a constant $T^{*} \in\left[\frac{\pi}{\sqrt{\kappa_{0}^{2}+\varepsilon}}, \frac{2 \pi}{\sqrt{\kappa_{0}^{2}+\varepsilon}}\right)$.

Proof. From the previous lemma we obtain that $\mu$ has only isolated zeros. So Lemma 6.2 shows that the control follows the alternating pattern $u_{0} \vdash$ $-u_{0} \vdash u_{0} \vdash \ldots$, etc. And the time between successive switches is given by the formula of the previous lemma. 


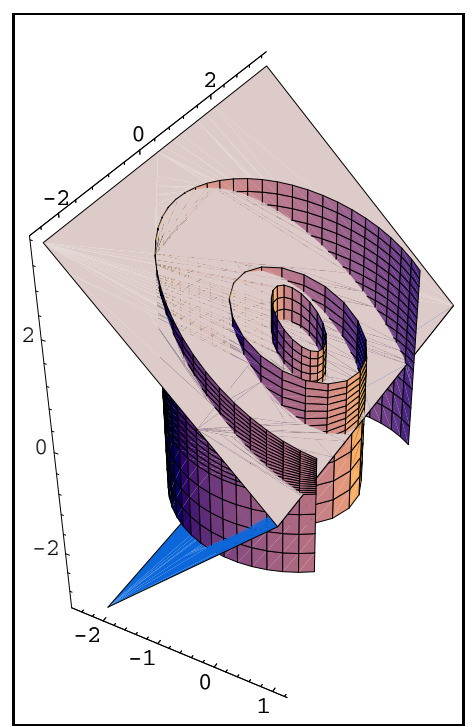

the surfaces

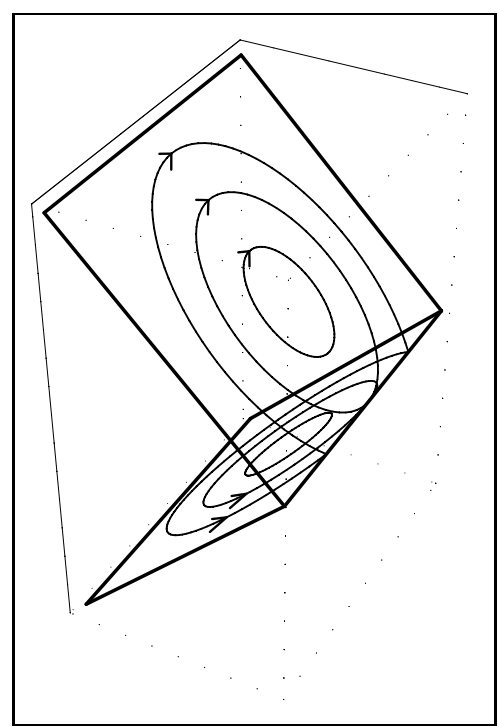

the flow

Figure 3. Coadjoint orbits and the flow of $p(t)$ in $\mathfrak{s e}(2)^{*}$ for $\lambda=1$.

Corollary 6.6. If $(g, p)$ is an extremal with $J_{3}=0$ and $J_{2}=\lambda=1$, and if the corresponding arc $x$ is optimal, then it is of type CLC.

Proof. The control $u$ follows the pattern $u_{0} \vdash 0 \vdash u_{1} \vdash 0 \vdash u_{2} \ldots$, and by Lemma 6.4 the time between two ' 0 's is exactly a full period of $X_{u_{i}}$. So the corresponding arc is CLCLC ..., where each intermediate circle is a full circle. Hence an optimal arc of that type has at most one geodesic segment, i.e. it is of type $C L C$.

This finishes the proof of Theorem 5.4.

REMARK 6.7. Even though integration of the ODE for $\mu$ is not difficult, we refer to [15, Corollary 4.5.1] for the formulas (in the special case $\kappa_{0}=1$ ). The really interesting geometric information is to be found in the covector $p(t) \in \mathfrak{g}^{*}$. W.1.o.g. we only look at $\left(h_{1}, h_{2}, H_{12}\right)$ - this corresponds to the two-dimensional problem. The level surfaces of $J_{2}=h_{1}^{2}+h_{2}^{2}+\varepsilon H_{12}^{2}$ are essentially the orbits of the coadjoint action of $G$ on $\mathfrak{g}^{*}$. In $\mathfrak{s e}(2)^{*}$ these are cylinders around the (vertical) $H_{12}$-axis, in $\mathfrak{s o}(3)^{*}$ these are spheres, and in $\mathfrak{s o}(1,2)^{*}$ these are one-sheeted hyperboloids, the upper or lower half of a two-sheeted hyperboloid or one half of a Lorentzian double cone (excluding the vertex at 0 ). The level surfaces of the optimal Hamiltonian

$$
\mathcal{H}(g, p)=h_{1}+\kappa_{0} \mu-\lambda \equiv 0, \quad \lambda \in\{0,1\},
$$

are the boundary of a (shifted) wedge in three-space with two faces. On each face the control is constant either $+\kappa_{0}$ or $-\kappa_{0}$. The switches occur when $p(t)$ hits the edge of this wedge. An alternating switch means that $p$ passes from the upper to the lower face (and vice versa). Projecting into the $\left(h_{1}, h_{2}\right)$-plane one can interpret the time between switches as the euclidian angle between the origin and the two intercepts of $p$ with the edge of the wedge. Figure 3 shows the coadjoint orbits and the flow of $p(t)$ in $\mathfrak{s e}(2)^{*}$. In ESAIM: COCV, FEBRUARY 1998, VOL. 3, 1-22 


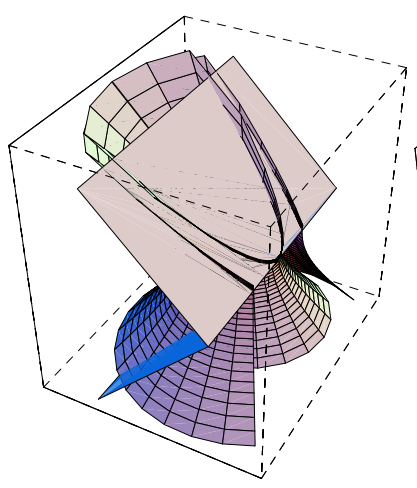

$\kappa_{0} \leq 1$

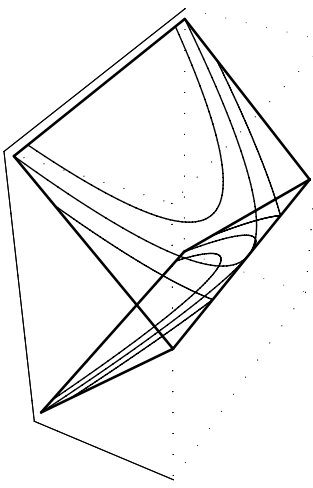

the flow

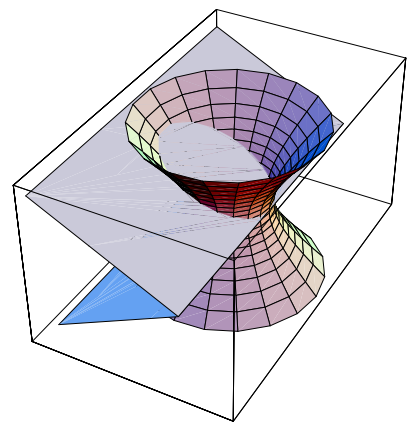

$\kappa_{0}>1$

Figure 4. Coadjoint orbits and the flow of $p(t)$ in $\mathfrak{s o}(1,2)^{*}$ for $\lambda=1$.

$\mathfrak{s o}(3)^{*}$ one has to replace the cylinders by spheres, but the flow essentially looks the same.

In the hyperbolic case the geometric difference between the noncontrollable and the controllable case is neatly visualized. If $\kappa_{0} \leq 1$, then the intersection of $\mathcal{H}=1$ with a one-sheeted hyperboloid is never closed - so $p(t)$ cannot hit the edge of the wedge twice. On the other hand if $\kappa_{0}>1$, this intersection is always a closed curve, as in the euclidian and spherical case. And the flow of $p(t)$ looks similar.

REMARK 6.8. Since both referees pointed out that these pictures bear a significant similarity with those in [7], a few more remarks are in order. Kupka's theory [7] cannot be applied to the problem under consideration for the following reason: the ODE (2.1) is affine in the controls and the cost functional $c(u) \equiv 1$ is independent of $u$. Therefore, if $\mu=\left\|H^{\prime}\right\|=0$, then every $v \in \mathcal{U}$ is a maximizing control. So the basic assumption that the set of maximizing controls is finite [7, Def. 1] is violated at all points of the switching manifold $\Sigma_{\lambda}=\left\{p \in \mathfrak{g}^{*} \mid H^{\prime}=0, h_{1}=\lambda\right\}$.

This problem may be overcome at those points of $\Sigma_{\lambda}$ where $J_{2}-\lambda^{2}>0$. Following the ideas in [13] we consider for $p_{0} \in \Sigma_{\lambda}$ only those maximizers $u^{*}$ for which $\overrightarrow{\mathcal{H}}_{u^{*}}\left(p_{0}\right)$ lies in the subtangent set at $p_{0}$ of the level set $\{p \mid \mathcal{H}(p)=\lambda\}$. If $J_{2}-\lambda^{2}>0$ at $p_{0}$, then this set of admissible maximizers consists of two points $u^{*},-u^{*}$, and the vectors $\overrightarrow{\mathcal{H}}_{ \pm u^{*}}\left(p_{0}\right)$ are transversal to $\Sigma_{\lambda}$, but only one of them is subtangent to $\{p \mid \mathcal{H}(p)=\lambda\}$. This is the geometrized version of Lemma 6.2. These points are in analogy to the normal switching points in [7].

There is only one more point left to discuss: $p^{*}=(\lambda, 0,0)$ with $\lambda=1$. In view of Lemma 5.2 it is clear that in Dubins' problem one also has to deal with singular arcs: a geodesic segment is generated by the control $u \equiv 0$ which is in the interior of the set $\mathcal{U}$ of admissible control values. Local theory for single-input systems with singular arcs can be found in [6], see in particular the discussion in Section 4.2.1 that actually covers the local behavior in the two-dimensional Dubins' problem. It would make sense to call $p^{*}$ an elliptic or hyperbolic fold point depending on whether $\kappa_{0}^{2}+\varepsilon>0$ 
or $\leq 0$. But this has no link with the classification of fold points in $[7$, p. 227$]$. Writing $\mathcal{H}_{u}$ instead of $\mathcal{H}_{X_{u}}$, etc., one computes for $u \neq v \in \mathbb{R}^{n-1}$, $\|u\|=\|v\|=\kappa_{0}$ that $\left\{\mathcal{H}_{u}, \mathcal{H}_{v}\right\}\left(p^{*}\right)=\left\langle p^{*},\left[X_{u}, X_{v}\right]\right\rangle=0$ and

$$
\left\{\mathcal{H}_{u}\left\{\mathcal{H}_{u}, \mathcal{H}_{v}\right\}\right\}\left(p^{*}\right)=\left\langle p^{*},\left[X_{u}\left[X_{u}, X_{v}\right]\right]\right\rangle=-u^{T} v+u^{T} u=\kappa_{0}^{2}-u^{T} v>0,
$$

regardless whether $\kappa_{0}^{2}+\varepsilon>0$ or not.

The author would like to thank the referees for pointing out Kupka's references he was not acquainted with so far.

\section{REFERENCES}

[1] L.E. Dubins: On curves of minimal length with a constraint on average curvature and with prescribed initial and terminal positions and tangents, Am. J. Math., 79, $1957,497-516$.

[2] V. Guillemin, S. Sternberg: Symplectic techniques in physics, Cambridge University Press, 1984.

[3] V. Jurdjevic: Non Euclidean Elastica, Am. J. Math., 117, 1995, 93-124.

[4] V. Jurdjevic: Casimir elements and optimal control, in Geometry in nonlinear control and differential inclusions, B. Jakubczyk ed., Polish Academy of Sciences, Warsaw, Banach Center Publ, 32, 1995, 261-275.

[5] V. Jurdjevic: Geometric Control Theory, Cambridge University Press, 1997.

[6] A.J. Krener, H. Schättler: The structure of small-time reachable sets in low dimensions, SIAM J. Control Optimization, 27, 1989, 120-147.

[7] I.A.K. Kupka: Geometric theory of extremals in optimal control problems. I: The Fold and the Maxwell Case, Transactions of the AMS, 299, 1987, 225-243.

[8] I.A.K. Kupka: The ubiquity of Fuller's phenomenon, in Non-linear controllability and optimal control, H. J. Sussmann ed., Marcel Dekker, New York, 1990, 313-350.

[9] J.E. Marsden, T. Ratiu: Introduction to Mechanics and Symmetry, Springer, New York, 1994.

[10] D. Mittenhuber: Dubins' problem in $\mathbb{H}^{3}$, integration of abnormal extremals, in preparation.

[11] D. Mittenhuber: Dubins' problem in hyperbolic space, in Control Theory and its Applications, R. Sharpe ed., Can. Math. Soc. Conf. Proc. Ser., to appear.

[12] D. Mittenhuber: Dubins' problem in the hyperbolic plane using the open disc model, in Control Theory and its Applications, R. Sharpe ed., Can. Math. Soc. Conf. Proc. Ser., to appear.

[13] D. Mittenhuber: Applications of the Maximum Principle to Problems in Lie Semigroups, in Semigroups in Algebra, Geometry, and Analysis, K. H. Hofmann, J. D. Lawson and E. B. Vinberg. eds., de Gruyter, Berlin, 1995, 311-336.

[14] F. Monroy Pérez: Non-Euclidean Dubins' problem, in Control Theory and its Applications, R. Sharpe ed., Can. Math. Soc. Conf. Proc. Ser., to appear.

[15] F. Monroy Pérez: Non-Euclidean Dubins ' problem: A control theoretic approach, $\mathrm{PhD}$ thesis, University of Toronto, 1995.

[16] J.G. Ratcliffe: Foundations of Hyperbolic Manifolds, Springer, Berlin, New York, 1994.

[17] J.A. Reeds, L.A. Shepp: Optimal paths for a car that goes both forwards and backwards, Pacific Journal of Mathematics, 145, 1990, 367-393.

[18] H.J. Sussmann: Shortest 3-dimensional paths with a prescribed curvature bound, in Proceedings of the 1995 IEEE Conference on Decision and Control, 1995, 3306-3312. 